\title{
Kondo lattice model at half-filling
}

\author{
R. Nourafkan ${ }^{1}$ and N. Nafari ${ }^{2}$ \\ ${ }^{1}$ Department of Physics, Sharif University of Technology, \\ P.O.Box: 11155-9161, Tehran, Iran. \\ ${ }^{2}$ Institute for Studies in Theoretical Physics and Mathematics, \\ P.O.Box: 19395-5531, Tehran, Iran.
}

(Dated: November 30, 2018)

\begin{abstract}
The single- and two-channel Kondo lattice model consisting of localized spins interacting antiferromagnetically with the itinerent electrons, are studied using dynamical mean field theory. As an impurity solver for the effective single impurity Anderson model we used the exact diagonalization (ED) method. Using ED allowed us to perform calculations for low temperatures and couplings of arbitrary large strength. Our results for the single-channel case confirm and extend the recent investigations. In the two-channel case we find a symmetry breaking phase transition with increasing coupling strength.
\end{abstract}

PACS numbers: 71.10.-w, 71.10.Fd, 71.10.+h, 71.10.Hf 


\section{INTRODUCTION}

The Kondo lattice model $(\mathrm{KLM})^{\underline{1}}$, in its single- and two-channel forms, is one of the fundamental microscopic models for the description of heavy fermion materials $2,3,4,5$. This model consists of itinerant conduction electrons coupled to localized spins sitting on the sites of a crystal lattice. The coupling is represented as an on-site exchange interaction between this spin and the conduction electron spin density. This rather simple model gives rise to complex many body physics whose detailed understanding requires further investigation. The nature of the ground state of this model results from the interplay between magnetic Ruderman-Kittel-Kasuya-Yosida (RKKY) interaction ${ }^{6}$ among the localized spins and the Kondo effect screening of these spins. The polarization cloud of conduction electrons produced by a local moment may be felt by another local moment. This provides the mechanism for the RKKY interaction. On the other hand, the same polarization cloud can also form a singlet bound state with the local moment, when coupling is strong ${ }^{7}$. The RKKY interaction leads to a long-range ordered antiferromagnetic phase in two and three dimensions and the Kondo effect screening leads to a short-range spin correlations due to the formation of coherent Kondo spin singlets. There is a quantum phase transition between the two limiting phases upon changing the parameters of the model ${ }^{8}$. It is generally believed that the half-filled single-channel Kondo lattice model (SC-KLM) exhibits a Kondo insulator phase for large coupling strength, whereas for smaller coupling strength a phase transition to an antiferromagnetic state occurs ${ }^{9}$.

In contrast to the SC-KLM, which shows Fermi liquid behavior, the two-channel Kondo lattice model (TC-KLM) shows non-Fermi liquid behavior. By TC-KLM we means two identical species of non-interacting electrons coupled antiferromagnetically to localized electron spins. The materials which may display the TC-KLM are rare-earth or actinide inter-metallic compounds such as $\mathrm{CeCu}_{2} \mathrm{Si}_{2}$ and $\mathrm{UBe}_{13}$. In these compounds, the $f$-orbital of the $\mathrm{Ce}$ and U elements remain strongly localized, essentially retaining their atomic character. Thus, the sites containing $\mathrm{Ce}$ or $\mathrm{U}$ atoms often possess a magnetic moment obeying the first Hund's rule of maximization of the total $f$-electron spins. These localized moments interact with light conduction electron states contributed by surrounding ligands ${ }^{10}$. The single impurity case ,i.e, this model has a non-Fermi liquid ground-state $\underline{\underline{11}}$. Non-Fermi liquid behavior is the result of the inability of conduction electrons to screen the impurity spin. For weak 
coupling, one has a free spin one-half object scattering electrons in both channels resulting in the same logarithmically growing scattering as the temperature is lowered. However, as the coupling constant grows, the impurity spin, on the one hand, tends to form a singlet, and on the other hand, the symmetry of the problem forbids it to favor either one of the two channels to form that singlet. The other possibility is to make a linear superposition of a singlet with each channel, but this leaves the unbound spin of the spectator channel carrying a two-fold spin degeneracy. In this case, as it turns out, it would behave like a new spin-half impurity which in turn wants to undergo another Kondo effect. Less is known about TC-KLM. In a study using quantum Monte Carlo (QMC), Jarrell and coworkers 12 have examined the PM phase of this model and found non-Fermi liquid behavior at low temperatures. The existence of sign problem in their QMC simulation limited their access to very low temperatures and large coupling strengths.

The aim of this work is to provide a thorough analysis of the SC-KLM and TC-KLM at half filling. We have also studied the TC-KLM at quarter-filling. The quarter-filling case for the two-channel model is analogous to the half-filled case for the single-channel model in that there is one conduction electron per impurity spin leading to complete screening at strong couplings. Our results for the single-channel case showed the presence of the Kondo insulator. In the two-channel case, we find a symmetry breaking phase transition with increasing coupling strength.

The organization of the paper is the following: In Sec. II, the KLM is introduced. In subsections III.A and III.B, results on the PM and AFM are presented, respectively. The main portion of subsection III.A is devoted to the calculation of self-energies, obtained at several coupling strengths. Subsection III.B deals with magnetic ordering and Sec. IV contains our concluding remarks.

\section{MODEL}

The KLM Hamiltonian is defined by

$$
H=-t \sum_{\substack{i m \\ \sigma}}\left(c_{i m \sigma}^{\dagger} c_{i+1, m \sigma}+H . c .\right)+\frac{J}{2} \sum_{\substack{i m \\ \alpha \beta}} \boldsymbol{S}_{i} \cdot\left(c_{i m \alpha}^{\dagger} \boldsymbol{\sigma}_{\alpha \beta} c_{i+1, m \beta}\right) \text {. }
$$

Here, $m$ is the channel index, assuming two values $(m=1,2)$ for the two-channel systems. $t$ is the conduction electron hopping amplitude, taken to be the same in both bands, $c_{i m \sigma}^{\dagger}\left(c_{i, m \sigma}\right)$ 
creates (annihilates) an electron on lattice site $i$, with channel index $m$ and spin projection $\sigma=(\uparrow, \downarrow)$, and $\boldsymbol{\sigma}$ is a pseudo-vector represented by Pauli spin matrices. $\boldsymbol{S}_{i}$ is the spin operator of the localized $f$-electrons.

For solving this hamiltonian we employ the dynamical mean field theory (DMFT) $\underline{13}$ which is a powerful tool to investigate the nonperturbative regimes of strongly correlated systems. In DMFT, the lattice model is mapped onto an effective impurity problem subject to a self-consistency condition which contains the needed information about the original lattice. The method becomes exact in the infinite coordination limit. In order to map KLM onto an appropriate impurity model, we closely follow the treatment given in Ref. 19, i.e., we introduce fermion operators $f_{i \sigma}$ to represent the spin operator of the localized $f$-electron $\left(S_{i}=\frac{1}{2}\right)$ as $\boldsymbol{S}_{i}=\frac{1}{2} \sum_{\alpha, \beta} f_{i \alpha}^{\dagger} \boldsymbol{\sigma}_{\alpha \beta} f_{i \beta}$, where the $f$-operators satisfy the constraint $f_{i \uparrow}^{\dagger} f_{i \uparrow}+$ $f_{i \downarrow}^{\dagger} f_{i \downarrow}=1$ for all $i$.

In the treatment of the effective impurity problem, the numerical method often employed is the quantum Monte Carlo (QMC) simulation based on Hirsch-Fye (H-F) algorithm and to avoid the sign problem one considers the classical spins ${ }^{14}$. However, the importance of a fully quantum mechanical treatment of the local spins has been addressed by Kienert and Nolting $\frac{15}{\underline{1}}$. Recently, Werner and Millis $\frac{16}{}$ have developed the stochastic quantum Monte Carlo (SQMC) which is based on the stochastic evaluation of diagrammatic expantion of the partition function. Although, the SQMC is faster than H-F's and the sign problem is less severe, but their access to low temperatures is limited. To avoid such limitations, we have solved the effective impurity problem using exact diagonalization (ED) algorithm 17 .18 . The ED can handle the whole interaction and temperature regimes. Also, due to fully quantum mechanical treatment of the local spins, we correctly treat spin-flip processes. In the self consistency loop of DMFT we use, as it is often done, a semi-circular density of states of bandwidth $4 t$ (Bethe lattice).

\section{RESULTS}

In this section we present our results on SC-KLM and TC-KLM obtained by iteratively solving the equations appearing in DMFT. All calculations are done at $T=0$, unless otherwise is specified. Moreover, to obtain the paramagnetic (PM) solutions we suppress the magnetic order by averaging over spin up and spin down in each orbital. In this way, we 
have obtained both PM and antiferromagnetic (AFM) DMFT solutions of the KLM.

\section{A. Pramagnetic Phase}

In this subsection, we consider the behavior of KLM in paramagnetic phase. We first report results on SC-KLM. Our calculations reproduces the results of Werner and Millis 16 obtained via stochastic QMC. In addition, the use of ED allowed us to access lower temperatures and smaller frequencies. Fig. 1 shows the self-energies calculated for several $J$-values at zero temperature. As $\omega_{n} \rightarrow 0$, even for smallest $J$, the imaginary part of the self-energies

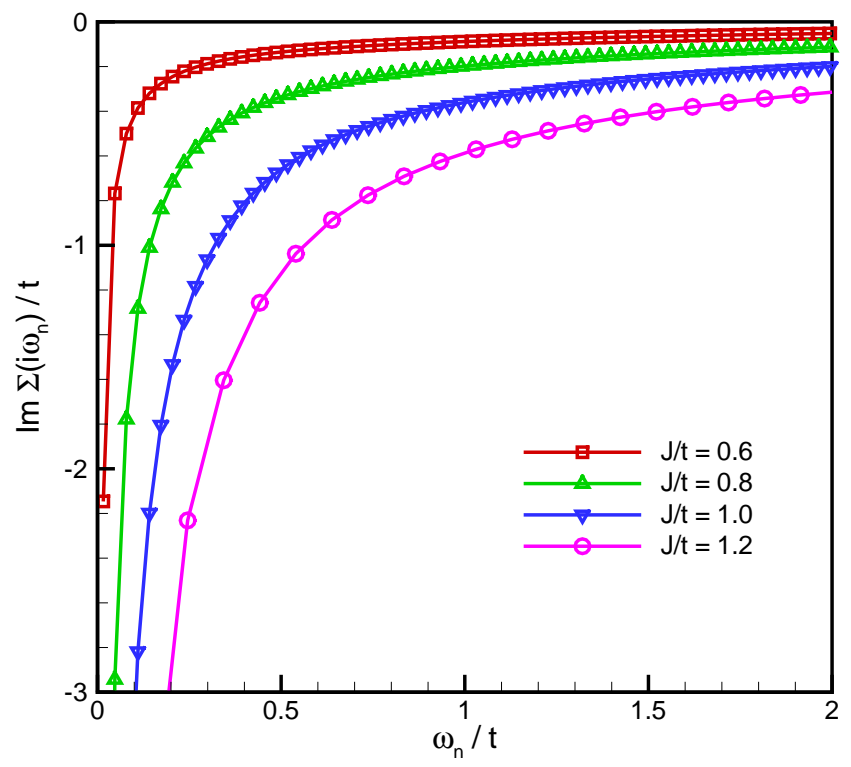

FIG. 1: (Color online) Imaginary part of the electron self-energy $\Sigma\left(i \omega_{n}\right)$ for the half-filled singlechannel Kondo lattice model at $T=0.0, \mathrm{~J} / t=0.6,0.8,1.0$ and 1.2.

diverges. This shows the presence of a charge gap at the half-filled SC-KLM and indicates that the system is in the Kondo insulating phase. This is a quantum disordered phase in which the conduction electrons are bound to local spins forming spin singlets. The local spin-local spin correlation function $\left\langle S_{z}(0) S_{z}(\tau)\right\rangle$ and the local spin-conduction spin correlation functions $\left\langle S_{z}(0) s_{z}(\tau)\right\rangle$ at $\beta t=200$ are shown in Fig. 2, The correlations decay rapidly with time, consistent with the formation of a gapped Kondo insulating state. Also the local spin-conduction spin correlation (inset) indicates an antiparallel alignment $\left\langle S_{z}(0) s_{z}(\tau)\right\rangle<0$. 


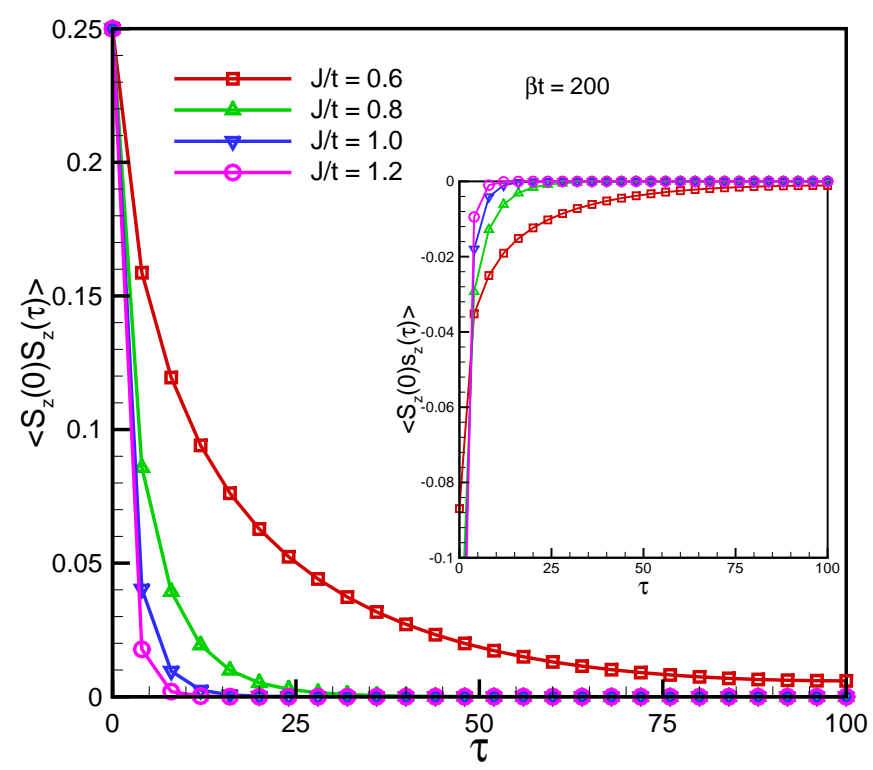

FIG. 2: (Color online) Imaginary time correlation function for the local moments calculated at half filling for the $J$-values as indicated and for $\beta t=200$. Inset shows conduction spin-local spin correlation functions. Both spin-spin correlation functions have exponentially decaying dependence on $\tau$.

Fig. 3 shows the dependence of the particle number per spin, $n$, on the chemical potential, $\mu$, for several $J$-values. This figure is similar to Fig. 7 of Ref. 16. However, because of the use of ED in our calculations, the gap in the excitation spectrum is quite evident for smaller values of $J / t$.

We next focus on TC-KLM. We have also calculated the imaginary part of self-energies for the two-channel case at quarter-filling and found that the situation in the two-channel model at quarter-filling is different from single-channel case at half-filling. It is believed that at the coupling strengths $J / t=0.20,0.60$ and 1.20 , electrons of different channels generate independent RKKY interaction between the localized moments. Fig. 4 shows the imaginary part of self-energies for TC-KLM at half-filling. In the left panel of Fig. 4 our results for small coupling strengths are shown, where the two bands show the same behavior. These results are in qualitative agreement with the corresponding results in Ref. 12. The right panel shows the result for larger coupling strengths. We see that with increasing coupling strength, a symmetry breaking phase transition occurs as shown in the right panel. As seen 




FIG. 3: (Color online) Density per spin plotted as a function of chemical potential for $\beta t=$ $200, J / t=0.2,0.6,0.8$ and 1.0. The data for $J / t=0.80$ and 1.0 are consistent with the opening of a charge gap.

in the figure, due to the fact that the self-energies assume two diffrenet values at a given Matsubara frequency, we have obtained two diffrent bands where at low frequencies one of them shows the metallic behavior and the other one diverges indicating an insulating phase. The inset of the right panel shows that at larger coupling strengths, conduction electrons of both channels form bound states with local spins.

\section{B. Magnetic Ordering}

We now study the magnetic ordering phenomena characteristic of the Kondo lattice. In the single channel KL we expect a quantum phase transition to a singlet phase for $J$ larger than a critical value. In Fig. 5 we show the staggered magnetization $m=n_{\uparrow}-n_{\downarrow}$ of the SC-KLM as a function of $J / t$ at $T / t=0.0,0.0125$. On the small $J$ side a strong temperature dependence is evident, reflecting the strong $J$ dependence of the Neel temperature at weak coupling. As one can see, by decreasing the temperature, the onset of magnetization shifts to lower $J$-values. At $J / t \geq 1$ the staggered magnetization rapidly drops to zero for either of 

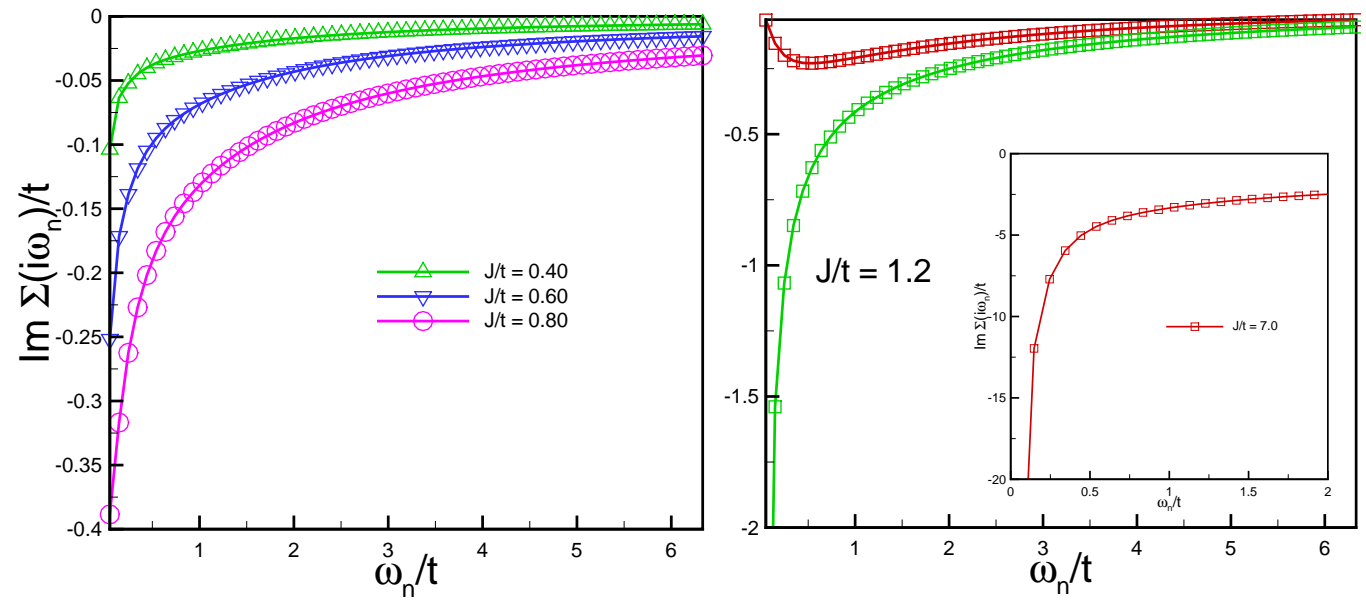

FIG. 4: (Color online) Imaginary part of the electron self-energy $\Sigma\left(i \omega_{n}\right)$ for the half-filled TC-KLM at $T=0.0$ and $J / t=0.2,0.4,0.6$ and 0.8 (left panel) and $T=0.0, J / t=1.2,7.0$ (right panel). The spontaneous symmetry breaking which takes place in $J / t \geq 1$ is obvious from the graph in the right panel. Further increase of the coupling strength caused the electrons in both bands to form bound states with local spins (see in the inset of right panel).

the two values of temperatures. This is the quantum phase transition to the singlet Kondo insulator phase. The right hand panel shows the staggered magnetization for TC-KLM. This figure shows the existence of an antiferromagnetic insulator for all physical coupling strengths.

\section{CONCLUDING REMARKS}

We have studied the single- and two-channel KLM at quarter- and half-filling using ED/DMFT approach. Compared to other frequently used DMFT impurity solvers such as QMC, the ED has the advantage of accessing very low temperatures and strong couplings. Our results for the single-channel case showed the presence of a charge gap at the half-filling and indicates that the system is in the Kondo insulating phase. In the two-channel case we find a symmetry breaking phase transition with increasing coupling strength.

As mentioned in the introduction, the nature of the ground state of the KLM results from the interplay between the magnetic RKKY interaction among the localized spins and the Kondo effect screening of these spins. In fact, we would expect to observe a strong 

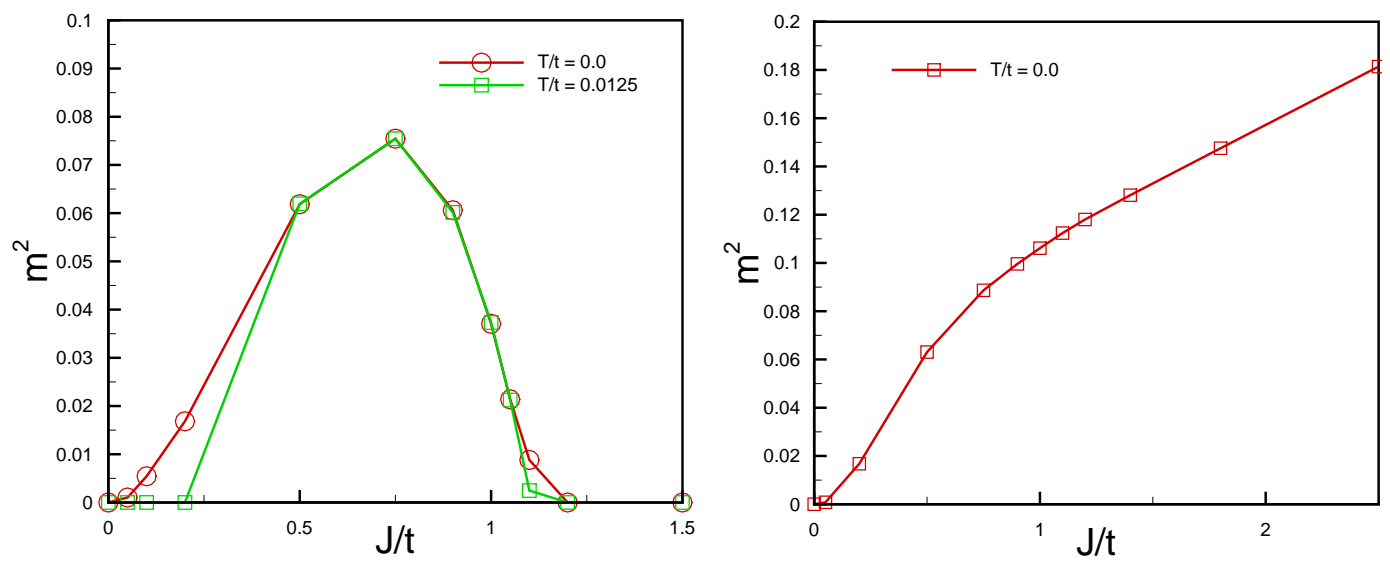

FIG. 5: (Color online) Staggered magnetization $m=n_{\uparrow}-n_{\downarrow}$ of the Kondo lattice model (half-filling, bipartite lattice). Left panel: staggered magnetization of single-channel Kondo lattice model as a function of $J / t$ for $T / t=0.0,0.0125$. There is an antiferromagnetic state at small coupling (for sufficiently low temperature) and a quantum phase transition to a paramagnetic insulator around $J / t=1.0$. Right panel: staggered magnetization of two-channel Kondo lattice model as a function of $J / t$ for $T / t=0.0$. There is an antiferromagnetic state for all physical coupling strengths.

competition between the RKKY interaction and the Kondo screening effect whenever the Néel and Kondo temperatures are rather close to each other.

Recent experiments in $\mathrm{CeIn}_{3}$ and $\mathrm{CePd}_{2} \mathrm{Si}_{2}$ exhibit such a strong competition as evidenced by their nearly the same Néel and Kondo temperatures. Moreover, these two heavy fermion compounds show, as we expect, an antiferromagnetic long range order $\underline{20}$ (See the Refs. therein). In order to investigate the properties of these systems, one might supplement the KLM with a Heisenberg hamiltonian for the localized spins, i.e., by including the antiferromagnetic exchange, $J_{A F}$, between core spins. In the future work, we plan to use such a modified model employing the more sophisticated cluster dynamical mean field theory. 


\section{Acknowledgments}

We wish to acknowledge useful discussions with R. Asgari.

1 For reviews see M. Gulacsi, Adv. Phys. 53, 769 (2004); H. Tsunetsugu, M. Sigrist and K. Ueda, Rev. Mod. Phys. 69, 809 (1997).

2 P. A. Lee et al., Comm. Condens. Matter Phys. 12, 99 (1986).

3 G. Aeppli, and Z. Fisk, Comm. Condens Matter Phys. 16, 155 (1992).

4 G. R. Stewart, Rev. Mod. Phys. 73, 797 (2001).

5 H. v. Löhneysen et al, cond-mat/0606317.

6 M. A. Ruderman, and C. Kittel, Phys. Rev. 96, 99 (1954); T. Kasuya, Prog. Theor. Phys. 16, 45 (1954); K. Yosida, Phys. Rev. 106, 893 (1957).

7 A. C. Hewson, The Kondo Problem to Heavy Fermions, Cambridge Studies in Magnetism (Cambridge Universiy Press, Cambridge, 1997).

8 S. Doniach, Physica B 91, 231 (1977).

9 V. Dorin and P. Schlottmann, Phys. Rev. B 46, 10800 (1992).

10 D. L. Cox and M. Jarrell, J. Phys: Condens. Matter 8, 9825 (1996).

11 P. Nozières, and A. Blandin, J. Phys. (Paris), 41, 193 (1980).

12 M. Jarrell, H. Pang, D.L. Cox, and K.H. Luk, Phys. Rev. B 77, 1612 (1996); M. Jarrell, H. Pang, and D. L. Cox, Phys. Rev. Lett. 78, 1996 (1997).

13 A. Georges, G. Kotliar, W. Krauth, and M. J. Rozenberg, Rev. Mod. Phys. 68, 13 (1996).

14 N. Furukawa, J. Phys. Soc. Jpn. 63, 3214 (1994).

15 J. Kienert, and W. Nolting, Phys. Rev. B 73, 224405 (2006).

16 P. Werner, and A. Millis, Phys. Rev. B 74, 155107 (2006).

17 M. Caffarel and W. Krauth, Phys. Rev. Lett. 72, 1545 (1994).

18 We solve the effective impurity problem by truncating the sum on bath levels to a small number of terms $n_{s}$, so that the Hilbert space is small enough to use, e.g., the Lanczos algorithm to compute the $T=0$ Green's function. In all our calculations presented here for SC-KLM the convergence of turncation has been checked, and in the case of TC-KLM we have used four bath levels per impurity level $\left(n_{s}=8\right)$. To check the accuracy of this approximation we have 
repeated the evalution of our results with five bath levels per impurity level $\left(n_{s}=10\right)$ and found fairly good agrement with $n_{s}=8$.

19 D. Meyer, C. Santos and W. Nolting, J. Phys: Condens. Matter 13, 25315 (2001).

20 M. Acquarone and C. I. Ventura, cond-mat/0708.0023v1. 